\title{
Defining protective immunity to dengue virus
}

\author{
By \\ Jeewandara Mudiyanselage Kamal Chandima Jeewandara
}

Thesis submitted to the University of Sri Jayewardenepura for the award of the Degree of Doctor of Philosophy in Immunology on Defining protective immunity to dengue virus. 
"The work described in this thesis was carried out by me under the supervision of Prof Gathsaurie Neelika Malavige and Prof Graham Ogg. A report on this has not been submitted in whole or in part to any university or any other institution for another Degree/Diploma".

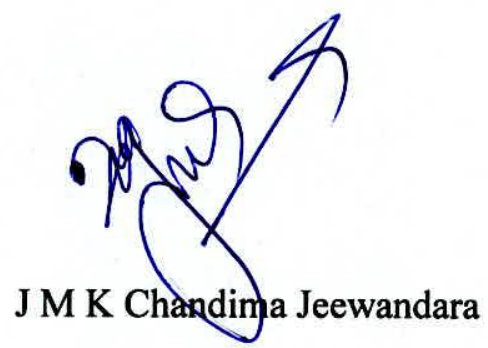

31 March 2015 
We certify that the candidate has incorporated all corrections, additions and amendments recommended by the examiners to this final version of the $\mathrm{PhD}$ thesis

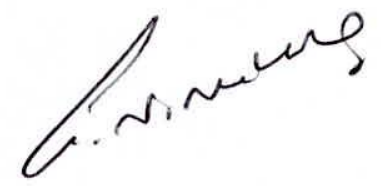

Prof Gathsaurie Neelika Malavige

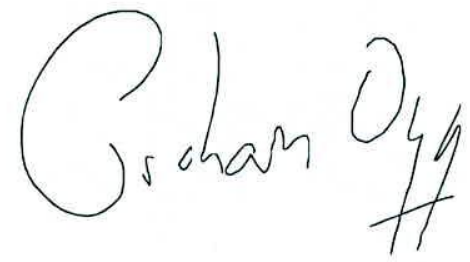

Prof Graham Ogg.

13 June 2016 
"We certify that the above statement made by the candidate is true and that this thesis is suitable for submission to the University for the purpose of evaluation".

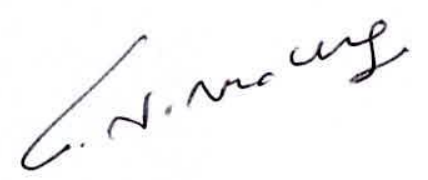

Prof Gathsaurie Neelika Malavige

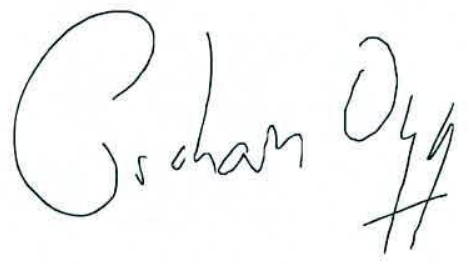

Prof Graham Ogg.

Date 17.6.2016 


\section{Table of contents}

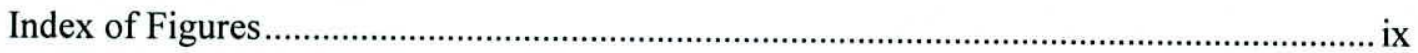

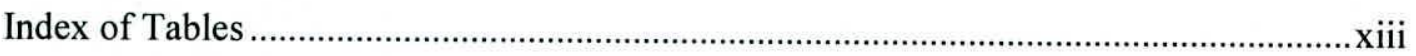

$1 \quad$ Introduction ................................................................................................................... 1

1.1 Dengue fever- Historical aspects ...........................................................................

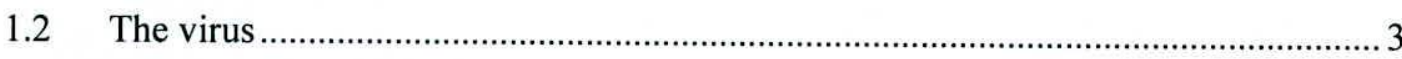

1.2.1 Virion morphology and functional biology ..................................................

1.2.2 Genomic Organization..........................................................................

1.2.3 NS proteins and their functions ............................................................... 6

1.2.4 Dengue virus genotypes .........................................................................

1.3 Immune response in dengue infections ................................................................

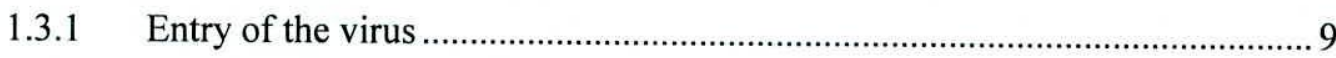

1.3.2 Innate immune response ........................................................................... 10

1.3.3 Adaptive immune response ..................................................................... 13

1.3.3.1 Antibody response ............................................................................. 13

1.3.3.2 Role of antibodies in enhancing DENV infection and disease .............. 15

1.3.4 T cell response in dengue infections ....................................................... 16

1.3.5 Role of cytokines in dengue infection ...................................................... 18

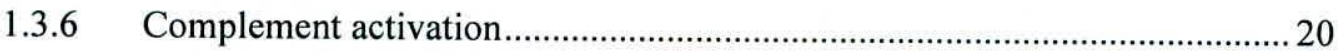




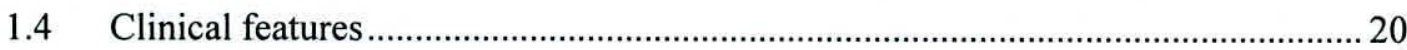

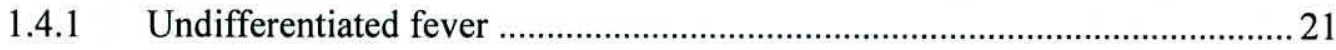

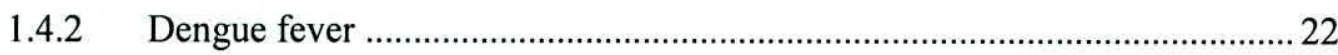

1.4.3 Dengue haemorrhagic fever (DHF) and dengue shock syndrome (DSS) .....23

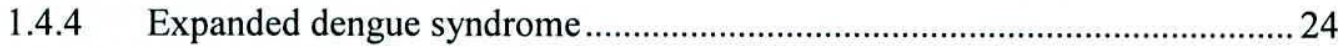

1.4.5 Convalescence and recovery in dengue ................................................... 24

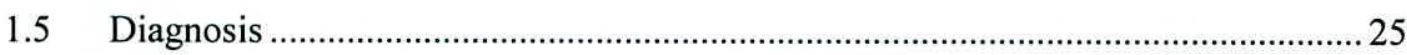

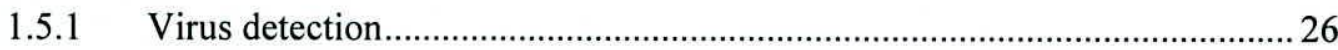

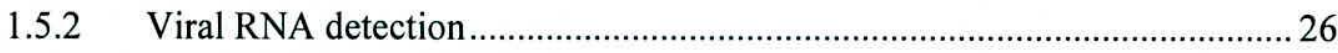

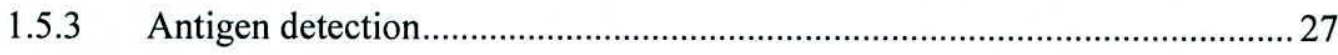

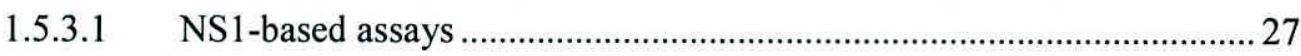

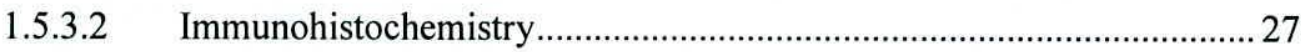

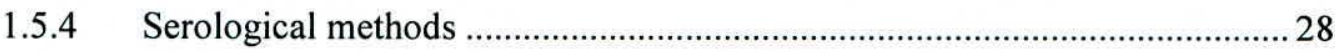

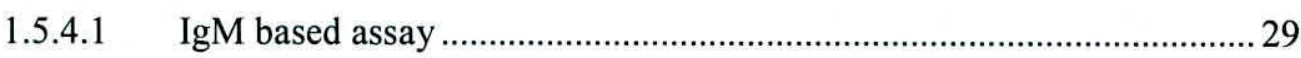

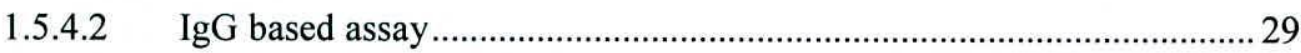

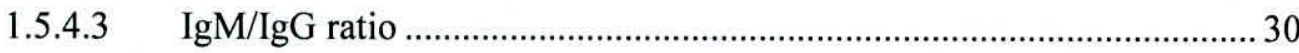

1.5.4.4 Haemagglutination/inhibition test ................................................... 30

1.5.4.5 Complement fixation test ............................................................... 30

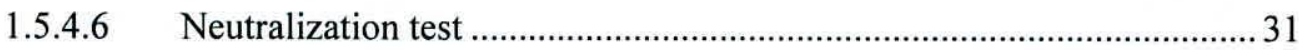




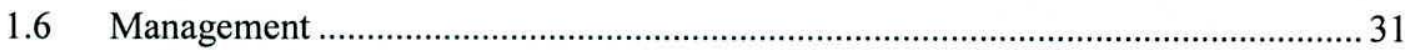

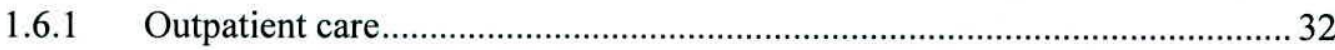

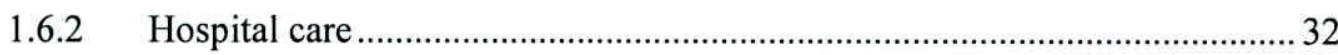

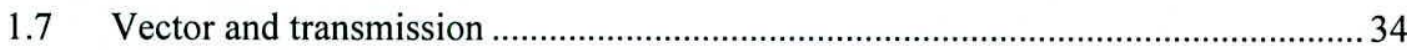

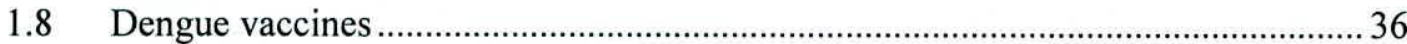

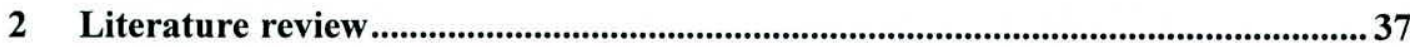

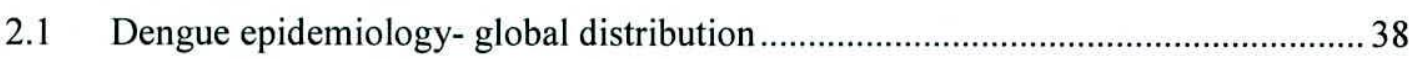

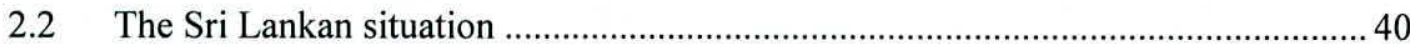

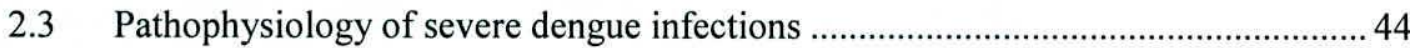

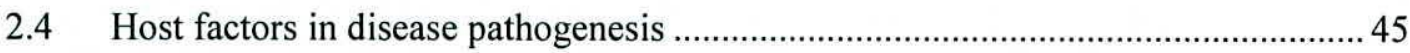

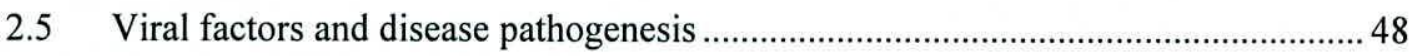

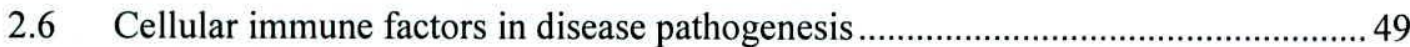

2.6.1 Antibody targets and functions .................................................................. 49

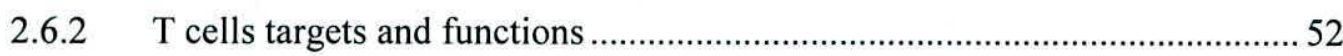

2.6.3 Memory $\mathrm{T}$ cell responses and cross reactive $\mathrm{T}$ cell responses ...................... 54

2.6.4 T cell responses in secondary infections …..............................................56

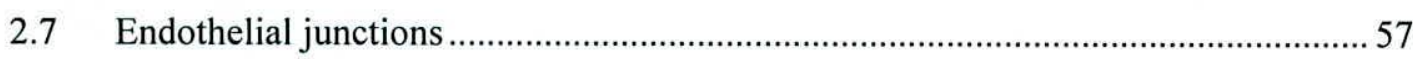

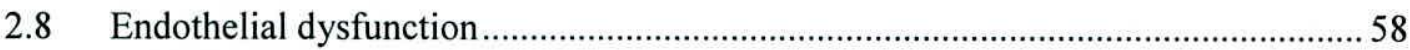

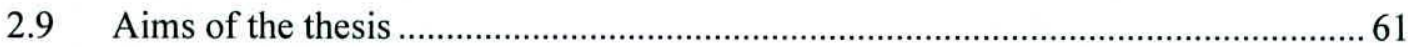




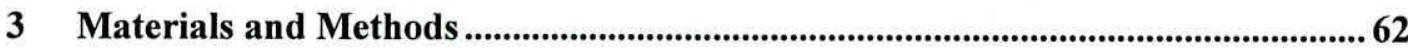

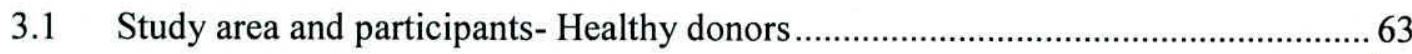

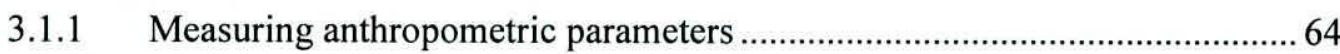

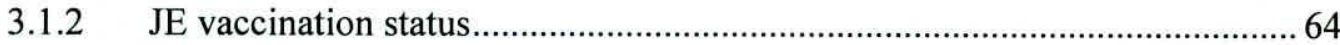

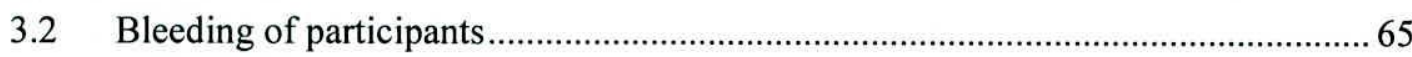

3.3 Separation of peripheral blood mononuclear cells (PBMCs) …................................66

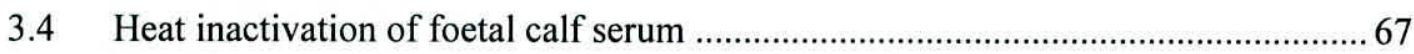

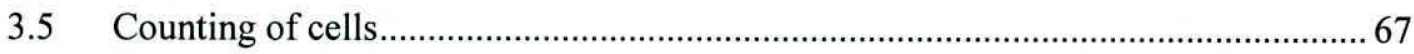

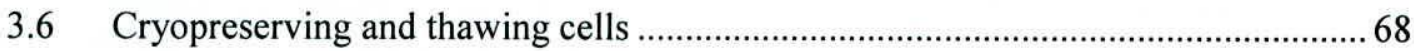

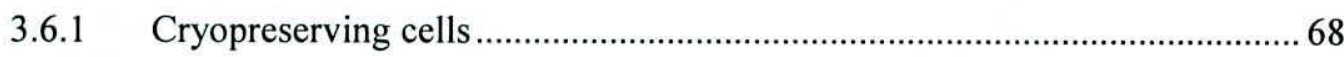

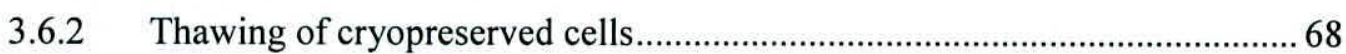

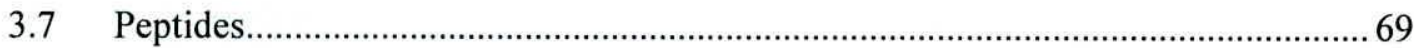

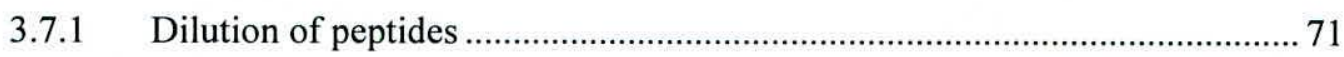

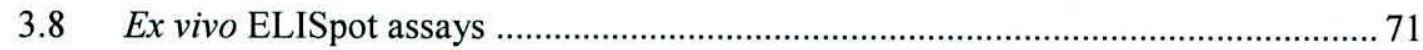

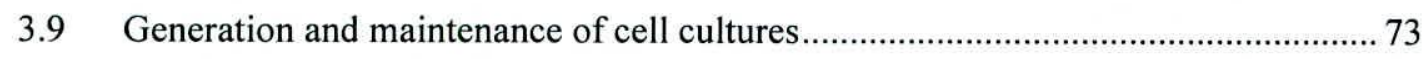

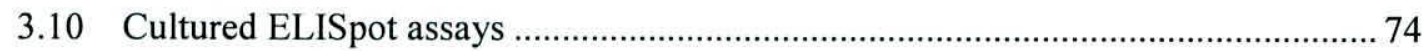

3.11 Serology

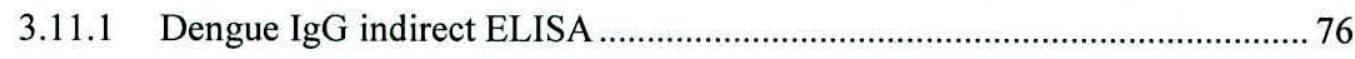

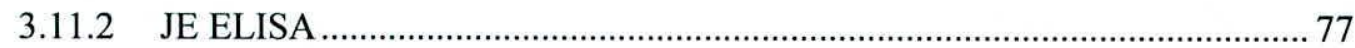




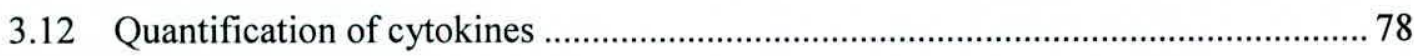

3.12.1 ELISA for granzyme B .......................................................................... 79

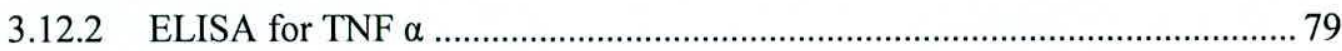

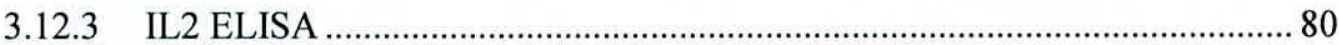

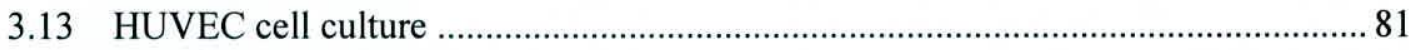

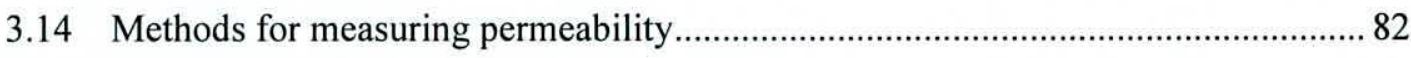

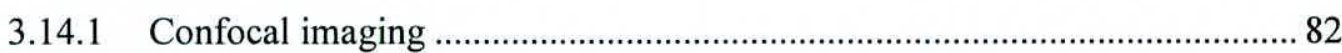

3.14.2 Immunofluorescence staining of ZO-1 …................................................ 83

3.14.3 Trans-endothelial electrical resistance (TEER) measurements ..................... 84

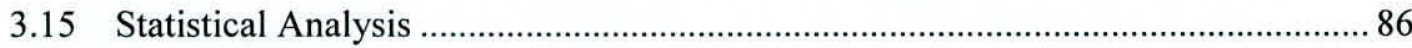

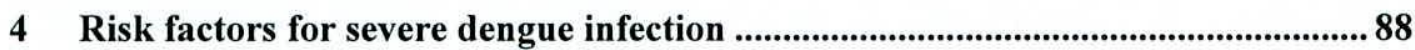

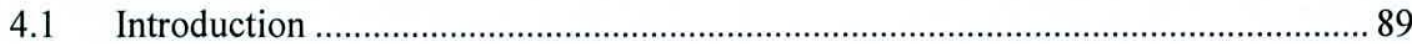

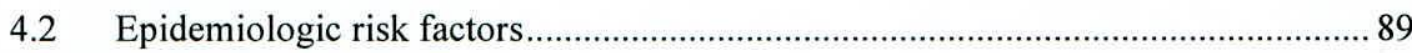

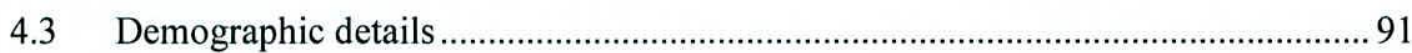

4.4 Relationship with co-morbidities and severe dengue in adults and children ...........94

4.5 Relationship with co-morbidities and severe dengue in children ............................. 97

4.6 Seroprevalence of dengue infections in the community......................................... 98

4.7 DENV antibody seropositivity and association with severe dengue ...................... 102

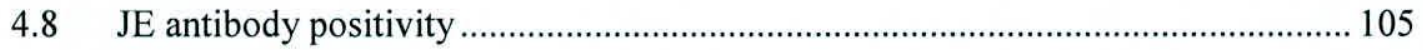


4.9 JE antibody seropositivity and association with severe dengue and JE vaccination....

4.10 Relationship between JE vaccination and dengue antibody positivity.

4.11 Relationship between JE vaccination and JE antibody positivity.

4.12 Relationship of DENV antibody response with number of past infections and serotype

4.13 Number of DENV infections and JEV seropositivity

4.14 Antibody titre of severe and subclinical dengue infections.

4.15 Discussion

5 Functionality of dengue virus specific memory $T$ cell responses

5.1 Functionality of the T cell responses in severe and subclinical infection 129

5.1.1 Functionality of the T cell responses to DENV-NS3 overlapping peptides 129

5.1.2 Functionality of the T cell responses to JEV vaccine.

5.2 Investigating the use of a $\mathrm{T}$ cell based assay to determine serotype-specific immune responses. 137

5.2.1.1 Usefulness of this assay in detecting seronegative patients for DENV 137

5.2.1.2 Cross reactivity of the assay with JEV vaccine 139

5.2.1.3 Usefulness of the assay in detecting past infection 140

5.2.2 Serotype-specific dengue immune responses and disease severity 145

5.3 Discussion. 151 
6 Role of platelet activating factor causing vascular leak in acute dengue infection..

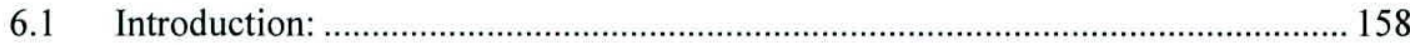

6.2 The effect of PAF on ZO-1 expression in HUVECs ........................................... 160

6.3 The effect of PAF on ZO-1 expression in HUVECs blocked by PAF receptor blocker

6.4 Determination of the ideal concentration of PAFRA 166

6.5 The effect of dengue patient serum on ZO-1 expression in HUVECs

6.6 The effect of dengue patient serum on ZO-1 expression in HUVECs pre-treated with PAF receptor blocker.

6.7 The effect of dengue patient serum on ZO-1 expression in HUVECs pre-treated with Rupatadine.

6.8 Effect of dengue sera on trans-endothelial resistance ….................................... 174

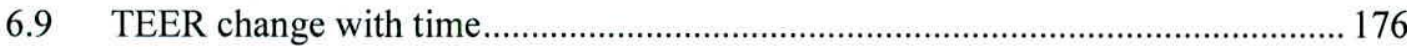

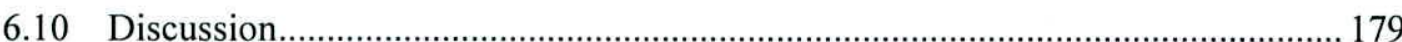

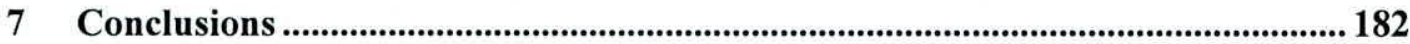

7.1 What differences were observed in the functionality of dengue virus specific memory $\mathrm{T}$ cell responses in individuals with past severe and subclinical dengue infection?.

7.2 What is the usefulness of the serotype specific peptide assay? 184 
7.3 What co-morbid factors contribute to severe disease dengue infection?

7.4 How does the immune response to a related flavivirus modulate clinical presentation and immune response to dengue virus?

7.5 What implications does JE vaccination have in Sri Lanka?

7.6 What is the age stratified seroprevalence of dengue infections in Colombo and does it change over time? 186

7.7 What is the role of lipid mediators in causing severe dengue infection? 187

7.8 Future directions

8 References

9 Appendices

9.1 List of publications

9.2 Communications from the thesis..

9.3 Awards and travel grants 225

9.4 Data collection forms 226 


\section{Index of Figures}

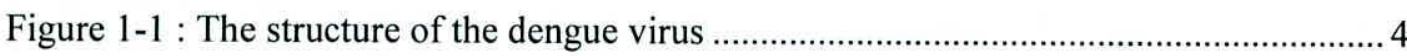

Figure 1-2 : Structure of the dengue virion and conformations of the E protein ................... 5

Figure 1-3 : The dengue virus genome

Figure 1-4 : Dengue viral infection and the immune response ............................................ 8

Figure 1-5 : Dengue virus lifecycle and subversion of the cellular antiviral response. ....... 11

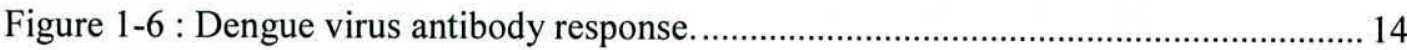

Figure 1-7 : Model for antibody-dependent enhancement of dengue virus replication ....... 15

Figure 1-8 : Cross reactive $\mathrm{T}$ cell response in dengue viral infections ............................... 17

Figure 1-9 : Clinical manifestations of dengue virus infection .......................................... 21

Figure 1-10 : Laboratory methods for the diagnosis of dengue infections..........................22

Figure 1-11 : Major diagnostic markers for dengue infection...........................................2. 28

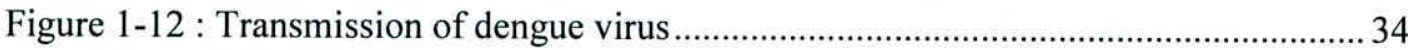

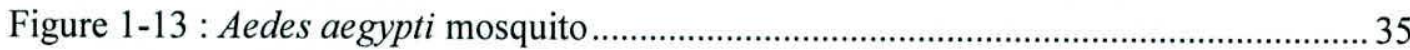

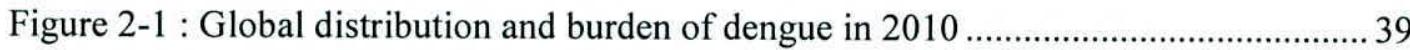

Figure 2-2 : Incidence of dengue cases and the CFR during 1989-2012 period................... 41

Figure 2-3 : Reported numbers of DHF cases in the year 2013 to the epidemiology unit Sri

Lanka

Figure 2-4 : Antibody responses to dengue virus protein targets and antibody functions. . 50

Figure 2-5: T cell responses to dengue virus protein targets and $\mathrm{T}$ cell effector functions 53

Figure 2-6 : Molecular organization of endothelial junctions 57 
Figure 3-1 : Diagrammatic representation of separation of layers following centrifugation

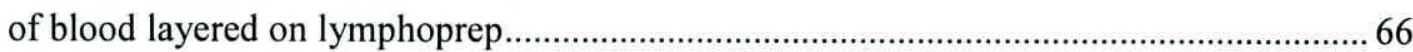

Figure 3-2 : Schematic representation of an IFN $\gamma$ ELISpot. ............................................. 71

Figure 3-3 : Ex vivo ELISpot responses in four dengue patients ........................................ 73

Figure 3-4 : Cultured ELISpot layout and a sample response demonstrating positive, a

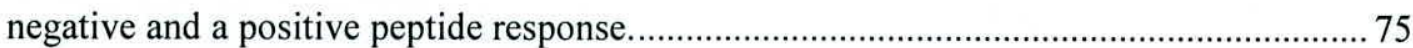

Figure 3-5 : Schematic representation of a dengue IgG ELISA......................................... 76

Figure 3-6 : Confocal imaging of the HUVEC cells demonstrating the ZO1 staining ....... 84

Figure 3-7 : Schematic representations of the TEER measurements ................................. 86

Figure 4-1 : Past dengue infections of the study cohort ........................................................98

Figure 4-2: The association of seropositivity for dengue and age in children ................. 100

Figure 4-3 : Correlation of DENV seropositivity with age in adults 101

Figure 4-4 : DENV antibody titres in children with past severe and sub clinical dengue infection

Figure 4-5 : DENV antibody titres of adults with past severe and sub clinical dengue infection

Figure 4-6 : Proportion of children who received the live and killed JE vaccine including those who did not complete and those who were not immunized 106

Figure 4-7 : Correlation of JEV seropositivity with age in children 108

Figure 4-8 : Correlation of JE seropositivity with age in adults

Figure 4-9 : JE antibody titre of individuals with past dengue and in dengue seronegative individuals 112 
Figure 4-10 : JE antibody titre of children who received JE live or killed vaccine 114

Figure 4-11 : DENV antibody titre and the number of dengue infections

Figure 4-12 : DENV antibody titre and infection with different DENV serotypes. 118

Figure 4-13:JE antibody titre and the number of past dengue infections

Figure 4-14 : JEV antibody titres in individuals who responded to different DENV serotypes

Figure 4-15: Serum IgG antibody titres of the severe secondary infections 122

Figure 4-16 : Serum IgG antibody titres of the primary infections

Figure 5-1 : Dengue-specific immune responses and disease severity 130

Figure 5-2: Cross reactive dengue-specific immune responses and disease severity. 135

Figure 5-3 : Cross reactive dengue-specific immune responses and disease severity. 136

Figure 5-4 : Response of serotype specific peptides to one seronegative individual before and after the DENV infection. 138

Figure 5-5 : Response of serotype specific peptides in seropositive individuals 141

Figure 5-6: The association of the number of DENV serotypes individual responded to with the age of the individual 146

Figure 5-7: The numbers of responses to DENV serotype specific peptides of those who had severe dengue and past sub clinical dengue infection 147

Figure 5-8 : The association of DENV-NS3 specific IFN $\gamma$-producing T cell responses with age. 149

Figure 5-9: Percentage of individuals who had severe dengue and sub clinical dengue infection who responded to different serotypes of DENV 150 
Figure 6-1 : Serum PAF levels in patients and healthy individuals

Figure 6-2 : ZO-1 expression in HUVECs with different concentrations of PAF and immunofluorescence staining.

Figure 6-3 : ZO 1 expression in HUVECs treated with different concentrations in PAF and pre-treated with a PAFRA 164

Figure 6-4 : Immunofluorescence staining of ZO 1 expression in HUVECs treated with different concentrations of PAF and pre-treated with a PAFRA. 165

Figure 6-5 : ZO 1 expression in HUVECs treated with different concentrations of PAF and pre-treated with different concentrations of PAFRA 167

Figure 6-6 : ZO 1 expression in HUVECs treated with different concentrations of PAF and DS and pre-treated with PAFRA 168

Figure 6-7 : Immunofluorescence analysis of tight junction protein ZO-1 in HUVECs. .. 170 Figure 6-8 : ZO 1 expression in HUVECs in DHF serum pre-treated with Rupatadine and control media and its immunofluorescence imaging 172

Figure 6-9: Change in the trans-endothelial electrical resistance with dengue sera and the effect of PAFRA and Rupatadine 175

Figure 6-10: Change in the trans-endothelial electrical resistance with dengue sera and the effect of PAFRA and Rupatadine over 24 hours. 177 


\section{Index of Tables}

Table 4-1 : Demographic characteristics of the adults in the study cohort ..........................92

Table 4-2 : Demographic characteristics of the children in the study cohort...................... 93

Table 4-3 : Presence of co-morbid factors and association with severity of dengue infection in adults

Table 4-4 : Presence of co-morbid factors and association with severity of dengue infection in children .97

Table 4-5 : Age stratified seroprevalence of DENV antibody in children 99

Table 4-6 : Age stratified seroprevalence of DENV antibody in adults .99

Table 4-7 : Age stratified seroprevalence of JE antibody in children 107

Table 4-8: Age stratified seroprevalence of JE antibody in adults 107

Table 4-9: Association of JE antibody positivity with past hospitalization due to dengue,

$\mathrm{JE}$ vaccination status and the type of JE vaccine given in children 110

Table 5-1 : Cytokine profiles of IFN $\gamma$, TNF $\alpha$, Granzyme B in individuals with Sub clinical dengue infection and severe dengue when PBMCs were stimulated with DENV-NS3 overlapping peptides or JE live vaccine. 132

Table 5-2 : Responses to the DENV serotype specific peptides of the 12 individuals at the time of recruitment and after they developed an episode of DHF during the study period

Table 6-1: Change in the trans-endothelial electrical resistance with dengue sera and the effect of PAFRA and Rupatadine over 24 hours. 178 


\section{List of abbreviations}

\begin{tabular}{|c|c|}
\hline $\mathrm{ADE}$ & Antibody-dependent enhancement \\
\hline CMV & Cytomegalovirus \\
\hline CFR & Case fatality rate \\
\hline DENV & Dengue virus \\
\hline DHF & Dengue haemorrhagic fever \\
\hline DNA & Deoxyribonucleic acid \\
\hline DSS & Dengue shock syndrome \\
\hline ELISpot & Enzyme-linked immunospot assay \\
\hline ELISA & Enzyme linked immunosorbent assay \\
\hline EBV & Epstein Barr virus \\
\hline FACS & Flurorescence-activated cell sorter \\
\hline FCS & Foetal calf serum \\
\hline FEC & Influenza virus, Epstein-Barr virus, and cytomegalovirus peptides \\
\hline $\mathrm{HCV}$ & Hepatitis $\mathrm{C}$ virus \\
\hline HIV & Human Immuno deficiency virus \\
\hline
\end{tabular}


HLA Human leukocyte antigen

HUVEC Human umbilical vein endothelial cells

IFN $\gamma \quad$ Interferon gamma

IL Interleukin

IgG Immunoglobulin G

IgM Immunoglobulin M

JEV Japanese encephalitis virus

MHC Major Histocompatibility Complex

NK Natural killer

NS Non-structural

NKT Natural killer T cells

PBMC Peripheral blood mononuclear cells

PBS Phosphate buffered saline

PCR Polymerase chain reaction

PHA Phytohaemagglutinin

PAF Platelet activating factor

PAFRA Platelet activating factor receptor antagonist 
PRNT Plaque reduction neutralization test

RNA Ribonucleic acid

RANTES Regulated on activation normal T expressed and secreted

SEM Standard error of mean

SD Standard deviation

SFU Spot forming units

SS Serotype specific

TEER Trans endothelial electrical resistance

TNF $\alpha \quad$ Tumor necrosis factor alpha

VEGF Vascular endothelial growth factor

VZV Varicella Zoster virus

WHO World health organization

ZO1 Zonula occludens 1

ZONAB ZO1-associated nucleic-acid binding 


\section{Acknowledgements}

The final thesis, the culmination of the long hard journey of my $\mathrm{PhD}$ would not have been achieved without the support of many individuals who contributed in numerous ways in different stages of my $\mathrm{PhD}$ research.

I am deeply indebted to my supervisor Prof Neelika Malavige for her elemental role in my $\mathrm{PhD}$. It is a great honour being her first $\mathrm{PhD}$ student. If it had not been her dedication a $\mathrm{PhD}$ in immunology, which requires lot of expenditure on research, would have been only a dream. She has been a wonderful teacher, a mentor, who constantly guided and assisted with her expertise in every step of the way. Her well-rounded mentorship helped me to grow as a research scientist. She encouraged me to be an independent thinker while providing valuable feedback. Her generosity with her expertise is remarkable and her enthusiasm for immunology has always been a motivation.

I am extremely grateful to Prof Graham Ogg, my external supervisor who kindly agreed to have me in Oxford as a student. It was a rare privilege to learn from a world-renowned professor in a world-class laboratory. I have been given the exposure to the most sophisticated technology in medical research. The value of experience and knowledge I gained in my training in Oxford is beyond measure. I owe a great debt to Prof Graham Ogg, not just for the opportunity to work in Oxford but for his constant feedback and guidance provided from the beginning to the end. 
My studies in Oxford which were an integral part of my research could be achieved only because of two organizations. I wish to express my sincere gratitude to the Association of Commonwealth Universities for granting me a Commonwealth Scholarship to pursue my postgraduate studies in a prestigious university and to the University of Oxford for granting me the studentship and providing excellent facilities to carry out my research work.

I consider myself extremely fortunate to have the University of Sri Jayewardenepura as my employer as I was well supported and encouraged during the course of my $\mathrm{PhD}$. I like to mention Dr N L A Karunathne- former Vice Chancellor of the University, Prof Mohan de Silva, former Dean of the Faculty of Medical Sciences, Prof Sampath Amarathunga, the Vice Chancellor and Prof Surangi Yasawardena, the Dean of the Faculty of Medical Sciences with extreme gratitude.

I have been able to balance the work of a lecturer and a student only because of the great support I received from the Department of Family Medicine of University of Sri Jayewardenepura. I am grateful for the head of the department and my fellow lecturers for being understanding and giving me the time I needed by sharing the duties and responsibilities among them.

The Department of Microbiology and its staff deserve a special mention for the support I received by them. I am grateful for the facilities I was supplied with and the support I received especially from the non-academic staff and the technical officers. 
I also thank the staff of the Centre for the Dengue Research for their valuable support, which contributed to the success of my research.

Throughout my $\mathrm{PhD}$, I was greatly supported by some fantastic research assistants who were very committed to their job. I wish to thank Thiruni Adikari, Laksiri Gomez, Dr Samitha Fernando, Dr Randika Fernando and Dr Shiran Paranavithane for being so supportive with such enthusiasm.

My colleagues Achala Kamaladasa, Mariyam Salimi, Danuta Gutowska-Owsiak and Anthony Cheung deserve a special thank for being such great colleagues by sharing knowledge and experience.

I like to thank my parents for all the years of hard work they put in to make the man I am today. I owe them for the good foundation they have given and the positive qualities they instilled

Finally, I thank my wife and son for bearing with me cheerfully during this challenging journey giving me the much needed motivation and encouragement. 


\title{
Defining protective immune responses to the dengue virus
}

\section{J M Kamal Chandima Jeewandara}

\begin{abstract}
Background: Although dengue infections can lead to severe clinical disease sometimes resulting in fatalities, the majority of both primary and secondary dengue infections result in mild/asymptomatic disease that is usually not diagnosed as dengue. Therefore, I proceeded to investigate epidemiological and co-morbid risk factors associated with severe dengue and the functionality of DENV specific memory $\mathrm{T}$ cell responses in relation to clinical disease severity. Furthermore, as platelet activating factor (PAF) is associated with an increase in vascular permeability in other diseases, I investigated its role in acute dengue infection.
\end{abstract}

Methods: 1689 healthy individuals were recruited. Information regarding their co-morbid illnesses, anthropometric measurements and Japanese Encephalitis vaccination status was recorded. The dengue and Japanese Encephalitis virus (JEV) antibody status was determined in all individuals. Using ex vivo IFN $\gamma$ ELISpot assays and by determining cytokines produced in ELISpot supernatants, I investigated the functionality of DENVspecific memory $\mathrm{T}$ cell responses in 338 individuals, who were naturally infected and either had severe dengue or had sub clinical dengue infection. PAF levels were assessed in 25 patients with acute dengue infection. The effect of dengue serum on tight junction 
protein ZO-1 was determined by using human endothelial cell lines (HUVECs). The effect of dengue serum on trans-endothelial resistance (TEER) and its reversibility in the presence of PAF receptor blocker was also measured on HUVECs.

Results: $1152 / 1689$ (68.2\%) individuals were seropositive for dengue however only $133 / 1152(9.8 \%)$ of them had been hospitalized due to dengue. A significant and positive correlation was observed for dengue antibody seropositivity and age in children (Spearmans $\mathrm{R}=0.9, \mathrm{p}<0.0001$ ) and in adults (Spearmans $\mathrm{R}=0.96, \mathrm{p}=0.004)$. Obesity, asthma, allergic rhinitis and a waist circumference of $>80 \mathrm{~cm}$ in women was significantly associated with increased risk of hospitalization for dengue. JEV antibody positivity was significantly associated with an increased risk of hospitalization in both adults $(\mathrm{p}<0.001)$ and in children $(\mathrm{p}=0.03)$. T cells of individuals with both past sub clinical dengue infection and severe dengue produced multiple cytokines when stimulated with DENV-NS3 peptides. DENV-NS3 specific T cells of those with sub clinical dengue infection were more likely to produce only granzyme $B(p=0.02)$, while those who had severe dengue were more likely to produce both TNF $\alpha$ and IFN $\gamma(\mathrm{p}=0.03)$ or TNF $\alpha$ alone.

PAF levels were significantly higher in patients with acute dengue $(n=25 ; p=0.001)$. Serum from patients with dengue significantly down-regulated expression of tight junction protein, ZO-1 $(p=0.004)$ in HUVECs. This was significantly inhibited $(p=0.004)$ by the use of a PAF receptor antagonist (PAFRA). Serum from dengue patients also significantly reduced TEER and this reduction was significantly $(\mathrm{p}=0.02)$ inhibited by prior incubation with the PAFRA. 
Conclusions: Obesity, asthma and JEV antibody positivity appear to be associated with a higher risk of hospitalization due to dengue virus. The types of cytokines produced by DENV-specific memory T cells appear to influence the outcome of clinical disease severity. The PAF is likely to be playing a significant role in inducing vascular leak in acute dengue infection, which offers a potential target for therapeutic intervention. 